\title{
Sleep-Disordered Breathing and Cardiac Arrhythmias
}

\author{
Nobuhisa Hagiwara, MD
}

$\mathbf{S}$ leep-disordered breathing (SDB) is characterized by a cessation of air flow during sleep, and can be divided into 2 types: obstructive sleep apnea (OSA) and central sleep apnea (CSA). OSA is a common breathing disorder that affects approximately 3-5\% of the general middle-aged population in Western countries and in Japan. OSA is characterized by recurrent pharyngeal airway collapse and temporary cessation of breathing during sleep. OSA is a $\geq 10$-s pause in respiration associated with ongoing ventilatory effort. A diagnosis of OSA is accepted when a patient has an apnea-hypopnea index (AHI: number of apneas and hypopneas per hour of sleep) $>5$ and symptoms of excessive daytime sleepiness. ${ }^{1}$ OSA is a proven risk factor for hypertension, heart failure, coronary artery disease and stroke.

\section{Article p 1466}

In contrast, CSA is associated with absent respiratory drive and results from instability in the chemoreflex control of breathing. CSA is a $\geq 10$-s pause in ventilation with no associated respiratory effort. Cheyne-Stokes respiration is a form of CSA and is characterized by a crescendo-decrescendo pattern of breathing with a central apnea at the nadir of ventilatory effort. CSA is highly prevalent in patients with heart failure.

Cardiac arrhythmias have been described in association with SDB, including sinus bradycardia, atrioventricular block, and supraventricular and ventricular arrhythmias. ${ }^{2}$ These cardiac arrhythmias have been implicated in sudden cardiac death of patients with SDB. ${ }^{3}$

Cardiac arrhythmias are reportedly more frequent in persons with SDB and increase with the number of apneic episodes and the severity of the associated hypoxemia. Nocturnal arrhythmias have been shown to occur in up to $50 \%$ of SDB patients. ${ }^{4}$ Atrial fibrillation (AF), nonsustained ventricular tachycardia, and complex ventricular ectopy are more common in subjects with SDB. Compared with those without SDB, individuals with SDB have 4-fold odds of AF, 3-fold odds of nonsustained ventricular tachycardia, and almost twice the odds of complex ventricular ectopy, even after adjustment for age, sex, body mass index, and the prevalence of coronary artery disease.

Prolonged apnea and hypoxemia in SDB elicit the diving reflex, which results in cardiac vagal activation. This vagal activation elicits bradyarrhythmias, such as atrioventricular block and sinus arrest, in SDB patients, even in the absence of a cardiac conduction abnormality. Guilleminault et $\mathrm{al}^{2}$ report that $8-11 \%$ of 400 patients with SDB has sinus arrest lasting more than $2.5 \mathrm{~s}$ and second-degree atrioventricular block. In general, severe bradyarrhythmias occur in association with a severe degree of SDB. Therefore, the treatment of nocturnal bradyarrhythmias in the setting of SDB and normal conduction would consist of treatment of SDB by continuous positive airway pressure (CPAP).

$\mathrm{AF}$ is the most prevalent sustained cardiac arrhythmia and affects $1-1.5 \%$ of the world population. A cross-sectional study reported that a strong association exists between $\mathrm{AF}$ and OSA, independent of age, sex, hypertension, heart failure and, body mass index. ${ }^{5}$ Gami et a ${ }^{6}$ described a retrospective cohort study of 3,542 people who underwent complete polysomnography without a prior history of AF. They showed that for individuals younger than 65 years old, OSA (AHI $\geq 5$; hazard ratio, 2.18 ), a decrease in nocturnal oxygen saturation (per $0.5 \mathrm{U} \log$ change; hazard ratio 3.29 ) and body mass index (per $1 \mathrm{~kg} / \mathrm{m}^{2}$; hazard ratio 1.07) were independent predictors of incident AF.

In a comprehensive meta-analysis with a total of 3,995 patients, it was demonstrated that OSA is associated with an increased risk of $\mathrm{AF}$ recurrence after catheter ablation. Overall, patients with OSA have a $25 \%$ greater risk of AF recurrence after catheter ablation than those without OSA (hazard ratio 1.25). ${ }^{7}$ Indeed, a small prospective observational study of patients who underwent successful cardioversion for AF reported that untreated OSA was associated with an $82 \%$ risk of recurrence within 1 year, approximately twice the risk of recurrence in OSA patients who were on CPAP treatment. ${ }^{8}$

Individuals with severe SDB have 2-3-fold higher odds of complex ventricular arrhythmias than those without SDB, even after adjustment for potential confounders. ${ }^{4}$ Both OSA and CSA are often observed in chronic heart failure (CHF) patients. In this issue of the Journal, Yamada et $\mathrm{al}^{9}$ report that SDB induces cardiac electrical instability and increases the risk of fatal ventricular arrhythmias in patients with CHF. In our study, ${ }^{10} 71$ patients with CHF and an implantable cardioverter-defibrillator (ICD) who were followed up for 180 days after a sleep study were prospectively evaluated. The frequency of appropriate ICD therapy and the day-night pattern of ICD therapy were compared between patients with and without SDB. Appropriate ICD therapy occurred more frequently in patients with SDB $(43 \%)$ than in those without SDB $(17 \%$; $\mathrm{P}<0.029)$. In a multivariate analysis, the presence of SDB was an independent predictor of appropriate ICD therapy (hazard ratio 4.05). Moreover, the rate of total ICD therapy from midnight to 6 a.m. was significantly higher in patients with SDB. Gami et al ${ }^{3}$ also reported a significantly higher peak rate of sudden cardiac death of patients with SDB during sleep (midnight to 6 a.m.) than in patients without SDB, in whom the peak sudden

The opinions expressed in this article are not necessarily those of the editors or of the Japanese Circulation Society.

Received April 4, 2013; accepted April 4, 2013; released online April 25, 2013

Department of Cardiology, Tokyo Women's Medical University, Tokyo, Japan

Mailing address: Nobuhisa Hagiwara, MD, Department of Cardiology, Tokyo Women's Medical University, 8-1 Kawada-cho, Shinjuku-ku,

Tokyo 162-8666, Japan. E-mail: mhagi@hij.twmu.ac.jp

ISSN-1346-9843 doi:10.1253/circj.CJ-13-0444

All rights are reserved to the Japanese Circulation Society. For permissions, please e-mail: cj@j-circ.or.jp 


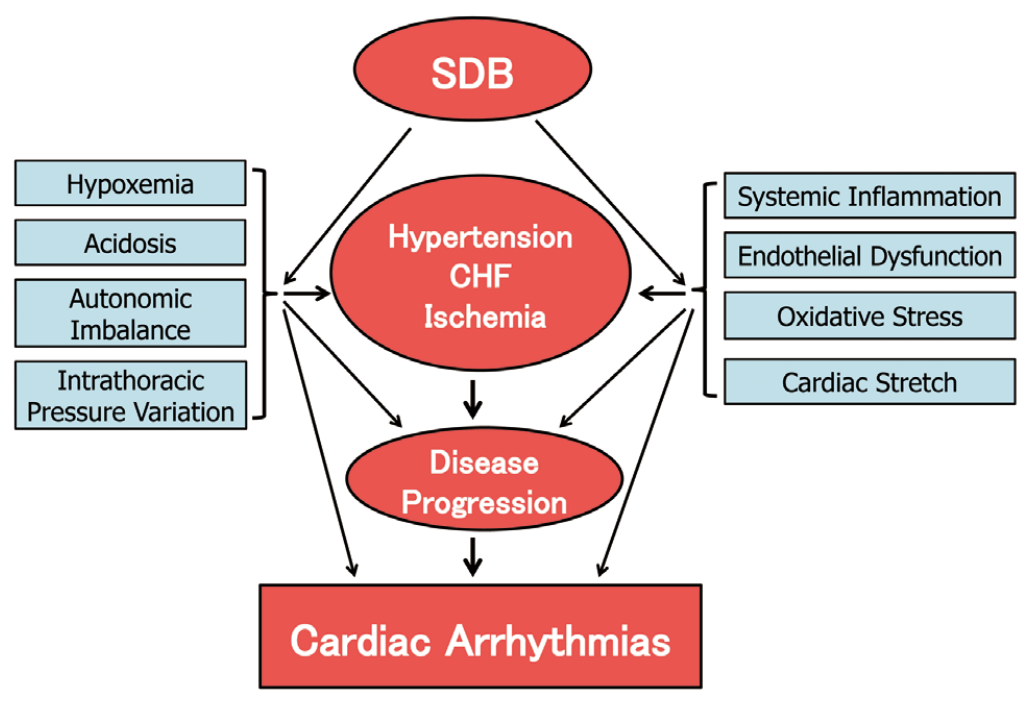

Figure. Mechanisms of cardiac arrhythmias in sleep-disordered breathing (SDB). $\mathrm{CHF}$, chronic heart failure.

death rate occurred later in the morning. These findings suggest that SDB may have a role in triggering sudden death during sleep, most likely through ventricular arrhythmias.

The exact mechanisms of the increased risk of cardiac arrhythmias are uncertain (Figure). However, the impairment of autonomic nervous control induced by SDB with CHF is thought to increasingly promote cardiac electrical instability and increase the frequency of ventricular arrhythmias. ${ }^{9}$ In humans, it is well-established that intermittent hypoxemia results in increased sympathetic tone. It is possible that SDB increases autonomic imbalance, intermittent apnea-induced hypoxia, and surges in left ventricular afterload elicited by repetitive cycles of sleep apnea, which are all likely to be important factors of increased ventricular arrhythmias in patients with CHF. Also, SDB causes hypoxemia, acidosis, arousal, vascular endothelial dysfunction, systemic inflammation, and elevated oxidative stress. These factors are known to be potent stimuli to myocardial ischemia, ${ }^{11}$ adverse cardiac remodeling and accelerated disease progression in patients with CHF. ${ }^{12}$ Recurrent airway collapse can result in significant variation in intrathoracic pressure and production of transmural force that contributes to heart chamber enlargement. The mechanical effects of SDB promote cardiac stretching, which may predispose to arrhythmias through a mechano-electrical feedback mechanism. ${ }^{13}$

Treatment of OSA may decrease the incidence of ventricular arrhythmias. In a randomized controlled trial of 1-month duration in patients with SDB and CHF, CPAP treatment resulted in a $58 \%$ reduction in the frequency of ventricular premature complexes during sleep and a parallel reduction in nocturnal urinary norepinephrine concentrations. ${ }^{14}$

There are no conclusive long-term clinical trials for the effect on cardiac arrhythmias of treating SDB. Therefore, it is suggested that large-scale and long-term randomized controlled trials are required to evaluate the efficacy of the treatment of SDB for preventing or treating cardiac arrhythmias using CPAP or adaptive servoventilation. ${ }^{15}$

\section{References}

1. Sleep-related breathing disorders in adults: Recommendations for syndrome definition and measurement techniques in clinical research: The Report of an American Academy of Sleep Medicine Task Force. Sleep 1999; 22: 667-689.

2. Guilleminault C, Connolly SJ, Winkle RA. Cardiac arrhythmia and conduction disturbances during sleep in 400 patients with sleep apnea syndrome. Am J Cardiol 1983; 52: 490-494.

3. Gami AS, Howard DE, Olson EJ, Somers VK. Day-night pattern of sudden death in obstructive sleep apnea. N Engl J Med 2005; 24: 1206-1214.

4. Mehra R, Benjamin EJ, Shahar E, Gottlieb DJ, Nawabit R, Kirchner HL, et al. Association of nocturnal arrhythmias with sleep-disordered breathing: The Sleep Heart Health Study. Am J Respir Crit Care Med 2006; 173: 910-916.

5. Gami AS, Pressman G, Caples SM, Kanagala R, Gard JJ, Davison $\mathrm{DE}$, et al. Association of atrial fibrillation and obstructive sleep apnea. Circulation 2004; 110: 364-367.

6. Gami AS, Hodge DO, Herges RM, Olson EJ, Nykodym J, Kara T, et al. Obstructive sleep apnea, obesity, and the risk of incident atrial fibrillation. J Am Coll Cardiol 2007; 49: 565-571.

7. Ng CY, Liu T, Shehata M, Stevens S, Chugh SS, Wang X. Metaanalysis of obstructive sleep apnea as predictor of atrial fibrillation recurrence after catheter ablation. Am J Cardiol 2011; 108: 47-51.

8. Kanagala R, Murali NS, Friedman PA, Ammash NM, Gersh BJ, Ballman KV, et al. Obstructive sleep apnea and the recurrence of atrial fibrillation. Circulation 2003; 107: 2589-2594.

9. Yamada S, Suzuki H, Kamioka M, Suzuki S, Kamiyama Y, Yoshihisa A, et al. Sleep-disordered breathing increases risk for fatal ventricular arrhythmias in patients with chronic heart failure. Circ J 2013; 77: $1466-1473$.

10. Serizawa N, Yumino D, Kajimoto K, Tagawa Y, Takagi A, Shoda M, et al. Impact of sleep-disordered breathing on life-threatening ventricular arrhythmia in heart failure patients with implantable cardioverter-defibrillator. Am J Cardiol 2008; 102: 1064-1068.

11. Mooe T, Franklin KA, Wiklund U, Rabben T, Holmstrom K. Sleep disordered breathing and myocardial ischemia in patients with coronary artery disease. Chest 2000; 117: 1597-1602.

12. Usui K, Parker JD, Newton GE, Floras JS, Ryan CM, Bradley TD. Left ventricular structural adaptations to obstructive sleep apnea in dilated cardiomyopathy. Am J Respir Crit Care Med 2006; 173: $1170-1175$.

13. Franz MR. Mechano-electrical feedback in ventricular myocardium. Cardiovasc Res 1996; 32: 15-24.

14. Ryan CM, Usui K, Floras JS, Bradley TD. Effect of continuous positive airway pressure on ventricular ectopy in heart failure patients with obstructive sleep apnoea. Thorax 2005; 60: 781-785.

15. Oldenburg O. Cheyne-Stokes respiration in chronic heart failure: Treatment with adaptive servoventilation therapy. Circ J 2012; 76: $2305-2317$. 